\title{
Combining benzo[d]isoselenazol-3-ones with sterically hindered alicyclic amines and nitroxides: enhanced activity as glutathione peroxidase mimics
}

\author{
Tamas Kálai, ${ }^{a}$ Govindasamy Mugesh, ${ }^{b}$ Gouriprasanna Roy, ${ }^{b}$ Helmut Sies, ${ }^{c}$ Zoltán Berente ${ }^{d}$ and \\ Kálmán Hideg*a \\ ${ }^{a}$ Institute of Organic and Medicinal Chemistry, University of Pécs, P. O. Box 99, H-7602, Pécs, \\ Hungary. E-mail: kalman.hideg@aok.pte.hu; Fax: +36-72-536219; Tel: +36-72-536221 \\ ${ }^{b}$ Department of Inorganic and Physical Chemistry, Indian Institute of Science, Bangalore, \\ 560012, India \\ ${ }^{c}$ Institut für Biochemie und Molekularbiologie I, Heinrich-Heine-Universität Düsseldorf, \\ Postfach 101007, D-40001, Düsseldorf, Germany \\ dInstitute of Biochemistry and Medical Chemistry, University of Pécs, P. O. Box 99, H-7602, \\ Pécs, Hungary
}

Received 13th July 2005, Accepted 8th August 2005

First published as an Advance Article on the web 31st August 2005

Benzo $[d]$ isoselenazol-3-ones $N$-substituted with sterically hindered diamagnetic and paramagnetic five- or six-membered nitroxides or their precursors, including ring-opened diselenides, exhibit synergism in glutathione peroxidase (GPx) activity.

\section{Introduction}

Glutathione peroxidases (GPx) are selenoenzymes that protect various organisms from oxidative stress by catalyzing the reduction of hydroperoxides at the expense of glutathione (GSH) (eqn 1$)^{1,2}$

$$
\mathrm{ROOH}+2 \mathrm{GSH} \stackrel{\text { GPx }}{\longrightarrow} \mathrm{ROH}+\mathrm{GSSG}+\mathrm{H}_{2} \mathrm{O}
$$

The GPx superfamily contains four types of enzymes, the classical cytosolic GPx (cGPx), phospholipid hydroperoxide GPx (PHGPx), plasma GPx (pGPx) and gastrointestinal GPx (giGPx), all of which require selenium in their active sites for catalytic activity. ${ }^{3-9}$ The biological role of these key enzymes in the antioxidant defense system comprises not only detoxification, by reducing an overproduction of hydroperoxides, but also the regulation of intracellular signalling pathways ${ }^{10}$ and enzyme activities, such as that of 5-lipoxygenase. ${ }^{11}$ To overcome the intrinsic difficulties associated with the use of an enzyme as a drug, a number of low-molecular-weight organoselenium mimics ${ }^{12-14}$ have been developed for the reduction of hydroperoxides, which include the well-known GPx mimic ebselen (1) ${ }^{15,16}$ (Scheme 1.) and related compounds having direct $\mathrm{Se}-\mathrm{N}$ bond, ${ }^{17-22}$ cyclic compounds without any direct $\mathrm{Se}-\mathrm{N}$ bond ${ }^{23,24} \alpha-$ phenylselenoketones, ${ }^{25}$ and diaryl diselenides having Se $\cdots \mathrm{N}$ or $\mathrm{Se}$.. O intramolecular interactions. ${ }^{26-30}$ Ebselen (1) is a nontoxic compound at pharmacologically active concentrations, because

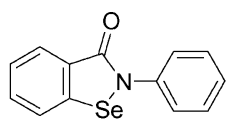

$1 v_{0}=12.16 \pm 0.94$

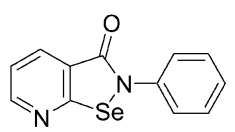

2

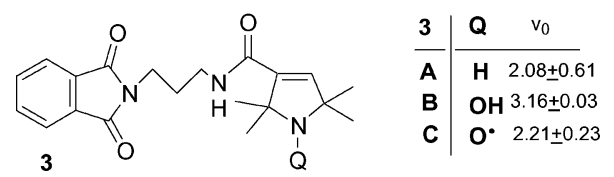

Scheme 1 Structure of ebselen 1, pyridine analogue of ebselen $\mathbf{2}$, cardioprotective compounds $\mathbf{3 A}-\mathbf{C}$ and observed initial reaction rates

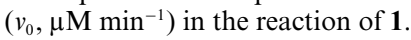

its selenium is not bioavailable. It is mostly bound to proteins in the form of selenyl sulfides ${ }^{15,16,31}$ and it is metabolized predominantly into glucuronidated species. ${ }^{32}$ Another important feature of ebselen is its inability to oxidize GSH in the presence of oxygen which normally leads to the uncontrolled production of superoxide and other free radical species. ${ }^{33}$ Although a number of attempts have been made to design and synthesize ebselen-related GPx mimics based on substituent effects or isosteric replacements, most of them met with limited success. For example, the replacement of the phenyl ring in ebselen by a pyridine ring (2) resulted in a complete loss of catalytic activity. ${ }^{20}$ In view of the potential applications of ebselen and related derivatives, we synthesized ebselen-based compounds by incorporating five- or six-membered $N$-heterocycles (as secondary amine connected to a diselenide "A form" or oxidized forms: $N$-hydroxyls "B form", $N$-oxyls " $\mathbf{C}$ form"). A number of non-selenium compounds having these substituents have been previously shown to posses cardioprotective activity. ${ }^{34}$ It has also been shown that pyrroline-based compounds such as $\mathbf{3 A}$ exhibit markedly enhanced protection against ischemia/reperfusioninduced myocardial contractile dysfunction ${ }^{35}$ and postischemic myocardial injury ${ }^{36}$ probably due to their combined antioxidative and antiarrhythmic activities because the in vivo oxidation of $\mathbf{3 A}$ to $\mathbf{3 C}{ }^{37}$

In this work, we report the GPx activity of a series of closely related benzisoselenazolones and show, for the first time, that the synergistic effect of selenium and pyrroline substituents enhances the antioxidant activity of these compounds.

\section{Results and discussion}

Compounds $\mathbf{1 2 C}-\mathbf{1 8 C}$ were synthesized using the method described earlier, ${ }^{38}$ i.e., by treating paramagnetic amines 5-11 in $\mathrm{CHCl}_{3}$ with 2-chloroselenyl-benzoylchloride $\mathbf{4}^{39}$ at ambient temperature in the presence of 2 eq. $\mathrm{Et}_{3} \mathrm{~N}$. Amines $5,{ }^{34} \mathbf{6},{ }^{40} 9,{ }^{41}$ $\mathbf{1 0}^{42}$ and $\mathbf{1 1}^{43}$ were prepared according to published procedures. Amine 7 was prepared by Suzuki coupling of paramagnetic vinylbromide $\mathbf{1 9}^{44}$ with 3-nitrobenzene boronic acid in the presence of $\mathrm{Ba}(\mathrm{OH})_{2}$ and $\mathrm{PdCl}_{2}\left(\mathrm{PPh}_{3}\right)_{2}$ as a catalyst in aq. dioxane followed by reduction of the resulted aromatic nitro compound 20 by Ehrenkaufer's method. ${ }^{45}$ Alkylation of paramagnetic 
Table 1 Initial rates $\left(v_{0}\right)$ for the reduction of $t-\mathrm{BuOOH}$ in the presence of catalysts ${ }^{a}$

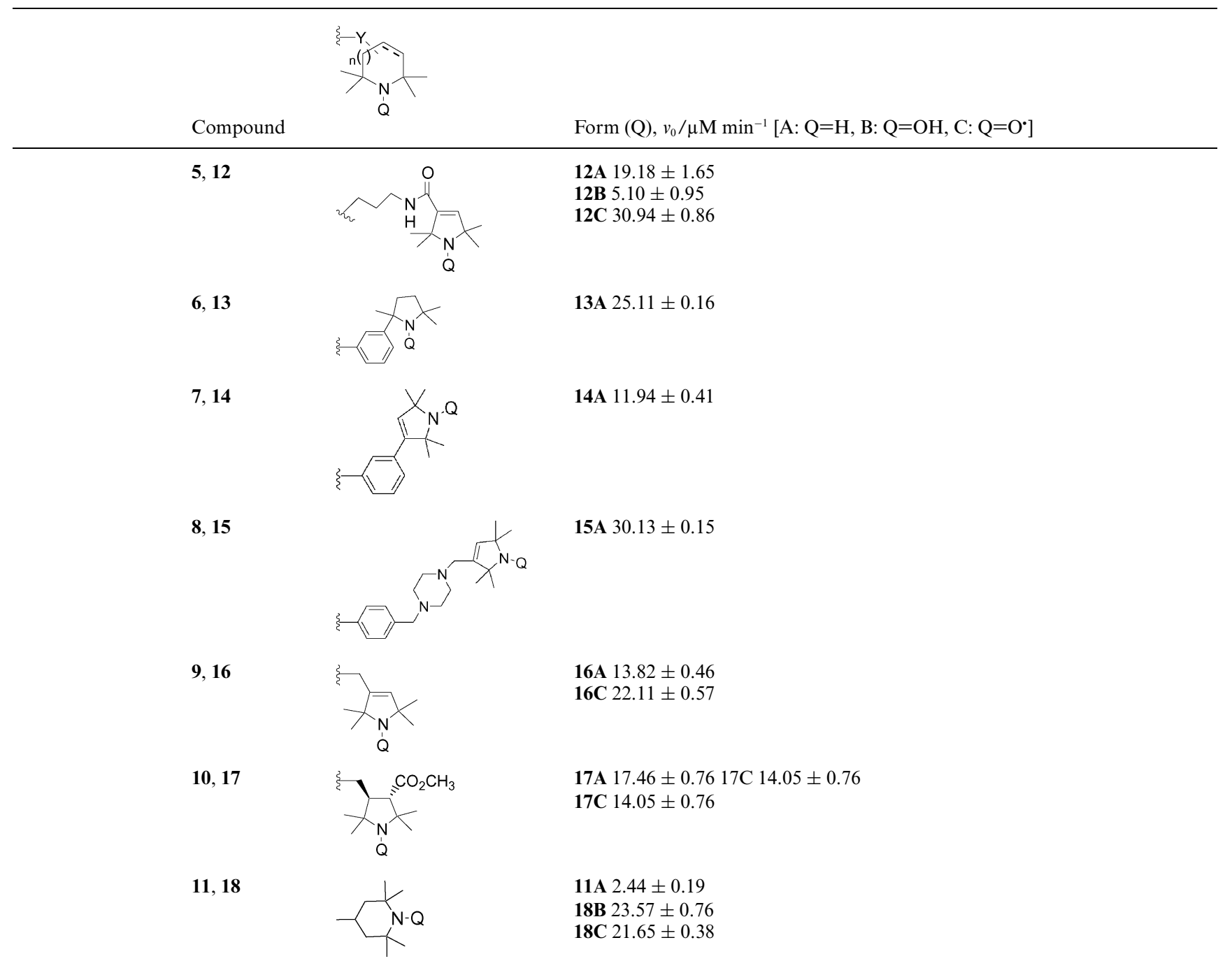

a Conditions: GSH: $1 \mathrm{mM}$; DTPA: $1 \mathrm{mM}$; GSSG reductase: 0.6 unit mL ${ }^{-1}$; NADPH: $0.1 \mathrm{mM}$; $t$-BuOOH: $1.2 \mathrm{mM}$; selenium catalysts: $0.05 \mathrm{mM}$; in $0.1 \mathrm{M}$ potassium phosphate buffer, $\mathrm{pH} 7.3, n=3$.

piperazine $\mathbf{2 1}^{46}$ with 4-nitrobenzylbromide afforded compound 22 the aromatic nitro group of which was reduced to yield amine $\mathbf{8}$ with ammonium formate in the presence of palladium on charcoal. The $N$-hydroxylamines 12-18 "B form" was achieved by refluxing the corresponding nitroxides 12-18 " $\mathrm{C}$ form" in EtOH saturated with $\mathrm{HCl}$ gas. ${ }^{35}$ Reduction of the "C form", a nitroxide with $\mathrm{Fe}$ powder in glacial acetic acid $^{47}$ yielded the corresponding secondary amine diselenides 12-18 "A form" with opening of isoselenazole ring (Scheme 2).

This ring opening takes place quite easily, even by reduction with ascorbic acid to selenol and oxidation during work-up or standing on air results in formation of a diselenide $\mathbf{2 3}$ (Scheme 3 ).

The ring opening followed by oxidation, e.g. structure of 12A18A compounds was observed by ${ }^{77} \mathrm{Se} \mathrm{NMR}$ measurements and data are in good agreement with earlier observations. ${ }^{48}$

The GPx activity of new compounds for reduction of $t$ $\mathrm{BuOOH}$ was screened spectrophotometrically at $340 \mathrm{~nm}$ as described earlier ${ }^{49}$ with minor modifications. It is evident from data in Scheme 1 and Table 1 that most of the selenium compounds used in the present study are more potent than ebselen (1). Interestingly, compounds $\mathbf{3 A}, \mathbf{3 B}$ and $\mathbf{3 C}$ which lack selenium in the benzene anellated five-membered ring and 11 nitroxide precursor, exhibited some GPx activity, however they are about ten-fold less active than isoselenazolone derivatives (Scheme 1). The pyrroline-substituted compounds showed remarkable GPx activity when attached to the basic benzoselenazolone unit. For example, the activity of compound 12C $\left(v_{0}=30.94 \pm 0.86 \mu \mathrm{M} \times \mathrm{min}^{-1}\right)$, in which both pyrroline and isoselenazole units are present, is higher than the sum of their activities in the individual cases $\left[v_{0}(1+3 C)=14.37 \pm\right.$ $1.17 \mu \mathrm{M} \times \mathrm{min}^{-1}$ ], suggesting a synergestic effect. The catalytic effect was demonstrated by changing the concentration of $\mathbf{1 2 C}$ and GSH (Table 1). The observed initial reduction rates $\left(v_{0}\right)$ were directly proportional to the catalyst concentration and rate increases with increasing concentration of GSH and with excess amount of it, the expected saturation kinetics were observed (figure not shown). We can find the same case in the catalytic activity of compound $\mathbf{1 8 C}$, which mimics the shape of ebselen with a six-membered ring connected to the nitrogen of isoselenasolone. This compound exhibits higher activity than ebselen (1) itself, confirming the role of the nitroxide based substituent. In other words, these two functionalities (isoselenazole and pyrroline/pyrrolidine/piperidine) individually show moderate effects on the reduction rate, however, when they are present together, the effect is supra-additive. The substitution pattern in the pyrroline ring also affects the reduction rate as observed for compounds $12 \mathrm{~A}$ and $13 \mathbf{A}$. Compound $\mathbf{1 3 A}$, in which the pyrroline ring is attached to the phenyl ring at the 2-position, exhibits 2-fold higher activity than the 3 -substituted 14A. The best initial rates were observed in the case of compounds with 

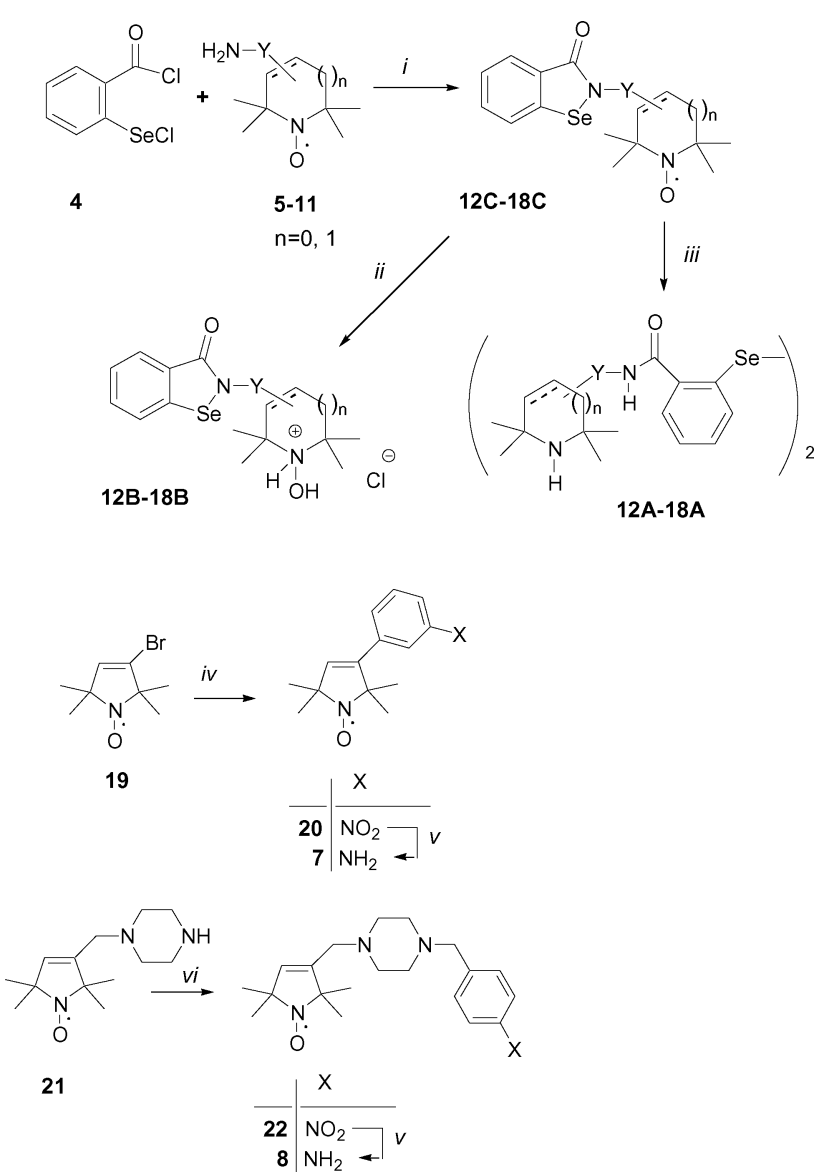

Scheme 2 . Reagents and conditions: (i) 4 (1.0 eq.), amine 5-11 (1.0 eq.), $\mathrm{Et}_{3} \mathrm{~N}$ (2.0 eq.), $\mathrm{CHCl}_{3}$, rt, $1 \mathrm{~h}, 35-73 \%$; (ii) $\mathrm{EtOH}-\mathrm{HCl}$, reflux, 20 min., 68-82\%; (iii) $\mathrm{Fe}, \mathrm{AcOH}, 70{ }^{\circ} \mathrm{C}, 1 \mathrm{~h}$, then $\mathrm{K}_{2} \mathrm{CO}_{3}, 47-59 \%$; (iv) 3-nitrophenyl boronic acid (1 eq.), $\mathrm{Pd}\left(\mathrm{PPh}_{3}\right)_{2} \mathrm{Cl}_{2}(5 \%), \mathrm{Ba}(\mathrm{OH})_{2}$ (1 eq.), dioxane-water, reflux, $3 \mathrm{~h}, 44 \%$; (v) $\mathrm{HCO}_{2} \mathrm{NH}_{4}$ (8 eq.), $\mathrm{Pd} / \mathrm{C}$, $\mathrm{MeOH}, 40{ }^{\circ} \mathrm{C}, 2 \mathrm{~h}, 35-43 \%$; (vi) 4-nitrobenzylbromide (1 eq.), $\mathrm{K}_{2} \mathrm{CO}_{3}$, $\mathrm{CHCl}_{3}$, reflux, $4 \mathrm{~h}, 68 \%$.

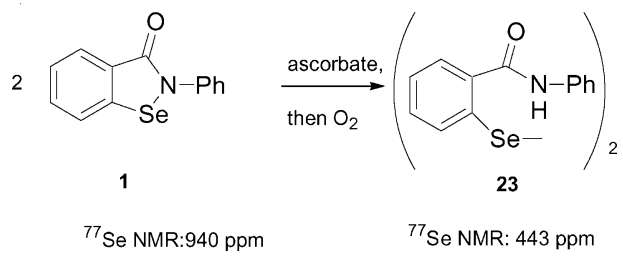

Scheme 3 Reduction of ebselen with ascorbic acid, followed by a spontaneous oxidation in an NMR tube.

a polar spacer group e.g. an amide for $\mathbf{1 2 C}$ and an amine for 15A. The combined catalytic effect appears to be less in the case of apolar spacers: 16A with a methylene group and 14A with a phenyl group as a spacer have less catalytic activity. It is interesting to note that compounds $\mathbf{1 8 B}$ and $\mathbf{1 8 C}$ without linking groups also show similar GPx activity as 16C. This indicates that the ring size of nitroxide heterocycle does not affect the reaction rate. Seemingly, the oxidation state of nitroxide moiety does not effect the initial reaction rate neither the isoselenzolone/diselenide form; $\mathbf{1 2 C}$ is more effective than $\mathbf{1 2 A}$, but as efficient as $\mathbf{1 5 A}$.

\section{Conclusions}

In conclusion, we have shown that due to the synergistic effect of selenium and amino functionalities the modification of the basic ebselen unit by introducing redox-active pyrroline groups greatly enhances the GPx activity of ebselen. The results presented here suggest a new concept that sterically hindered amino groups near the active site of GPx may act synergistically with selenium during the reduction of hydroperoxides. Compounds like 12-18 with broad antioxidant activity may serve as "ROS and RNS sponges" and can be promising candidates in future therapy of free radical mediated diseases.

\section{Experimental}

\section{General}

Melting points were determined with a Boetius micro melting point apparatus and are uncorrected. Elemental analyses (C, H, N, S) were performed on Carlo Erba EA 1110 CHNS elemental analyser. The IR (Specord 85) spectra were in each case consistent with the assigned structure. Mass spectra were recorded on a VG TRIO-2 instrument in the EI mode $(70 \mathrm{eV}$, direct inlet), the source temperature was $210{ }^{\circ} \mathrm{C}$, or an with Automass Multi instrument in the EI mode $(70 \mathrm{eV}$, direct inlet). ESR spectra were obtained from $10^{-5}$ molar solutions $\left(\mathrm{CHCl}_{3}\right)$, using an MS200 (Magnettech GMBH, Berlin) spectrometer. All radicals exhibited three equally spaced lines with $a_{\mathrm{N}}=$ 15.1-15.5 G. ${ }^{77} \mathrm{Se}$ NMR spectra were obtained on either a Bruker AVACE400 NMR spectrometer in $\mathrm{CDCl}_{3}-\mathrm{MeOH}(1$ : 1) mixture or on a Varian INOVA $400 \mathrm{WB}$ instrument and chemical shifts are reported with respect to $\mathrm{Me}_{2} \mathrm{Se}$. To obtain high resolution ${ }^{77} \mathrm{Se}$ NMR spectra of $\mathbf{1 2 C}$ it was reduced with the excess of co-dissolved $(\mathrm{PhNH})_{2}$. Ebselen and ascorbic acid reaction was recorded in $\mathrm{CDCl}_{3}-\mathrm{DMF}(1: 1)$ mixture. ${ }^{1} \mathrm{H}$ and ${ }^{13} \mathrm{C}$ NMR spectra were recorded with a Varian INOVA $400 \mathrm{WB}$ spectrometer at $400 \mathrm{MHz}$ at $25^{\circ} \mathrm{C}$, chemical shifts are given in ppm. Preparative flash column chromatography was performed on Merck Kieselgel $60(0.040-0.063 \mathrm{~mm})$. Qualitative TLC was carried out on commercially prepared plates $(20 \times 20 \times$ $0.02 \mathrm{~cm})$ coated with Merck Kieselgel $\mathrm{GF}_{254}$. Compounds

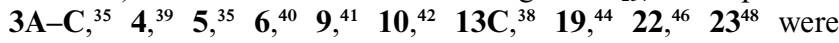
prepared according to published procedures, compound 1 was purchased from Calbiochem, compound 11A and other reagents were purchased from Aldrich. The GPx activity was followed spectrophotometrically at $340 \mathrm{~nm}$ on a Perkin-Elmer Lambda 35 UV-VIS Spectrophotometer. The test mixture contained GSH $(1 \mathrm{~mm})$, DTPA $(1 \mathrm{~mm})$, glutathione disulfide reductase $(0.6$ unit $\left.\mathrm{mL}^{-1}\right)$, and NADPH $(0.1 \mathrm{~mm})$ in $0.1 \mathrm{M}$ potassium phosphate buffer, $\mathrm{pH}$ 7.3. GPx samples were added to the test mixture at room temperature and the reaction was started by the addition of tert-butyl hydroperoxide (1.2 $\mathrm{mM}$, final concentration). The initial reduction rates were calculated from the rate of NADPH oxidation at $340 \mathrm{~nm}$. Each initial rate was measured at least three times and calculated from the first $5-10 \%$ of the reaction by using $6.22 \mathrm{mM}^{-1} \mathrm{~cm}^{-1}$ as the extinction coefficient for NADPH. For the peroxidase activity, the rates were corrected for the background reaction between $t-\mathrm{BuOOH}$ and GSH.

Synthesis of 3-(3-nitrophenyl)-2,2,5,5-tetramethyl-2,5-dihydro$1 H$-pyrrol-1-yloxyl radical (20). A mixture of compound 19 $(1.1 \mathrm{~g}, 5.0 \mathrm{mmol}), \mathrm{Ba}(\mathrm{OH})_{2} \cdot 8 \mathrm{H}_{2} \mathrm{O}(1.57 \mathrm{~g}, 5.0 \mathrm{mmol}), 3-$ nitrophenylboronic acid $(830 \mathrm{mg}, 5.0 \mathrm{mmol})$ and $\mathrm{PdCl}_{2}\left(\mathrm{PPh}_{3}\right)_{2}$ (140 mg, $0.2 \mathrm{mmol})$ in dioxane-water $(24 \mathrm{~mL}: 6 \mathrm{~mL})$ was stirred and refluxed for $3 \mathrm{~h}$. After cooling the dioxane was evaporated off, the aqueous phase was extracted with $\mathrm{CH}_{2} \mathrm{Cl}_{2}(2 \times 20 \mathrm{~mL})$. The organic phase was washed with water $(20 \mathrm{~mL})$, separated, dried $\left(\mathrm{MgSO}_{4}\right)$, filtered and evaporated under reduced pressure and the residue was purified by flash column chromatography (hexane-EtOAc) on silica gel to give compound $\mathbf{2 0}$ as a yellow solid $574 \mathrm{mg}(44 \%), \mathrm{mp} 69-71^{\circ} \mathrm{C}$. $R_{\mathrm{f}}: 0.50$ (hexane-EtOAc, 2 :

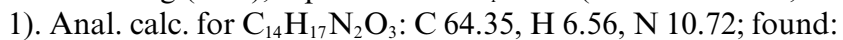
C 64.18, H 6.51, N 10.64\%. MS (EI): $m / z(\%) 261\left(\mathrm{M}^{+}, 28\right), 246$ (100), 231 (53), 216 (25), 128 (37).

\section{General procedure for Ehrenkaufer reduction $(7,8)$}

To a stirred solution of nitro compound $\mathbf{2 0}$ or $\mathbf{2 2}(5.0 \mathrm{mmol})$ and $\mathrm{HCO}_{2} \mathrm{NH}_{4}(2.52 \mathrm{~g}, 40 \mathrm{mmol})$ in $\mathrm{MeOH}(30 \mathrm{~mL})$ at $40^{\circ} \mathrm{C}$ 
under $\mathrm{N}_{2}$ atmosphere $120 \mathrm{mg} \mathrm{Pd} / \mathrm{C}(10 \%)$ was added and the mixture was stirred and refluxed until consumption of starting material (ca. $2 \mathrm{~h}$ ). After cooling, the mixture was filtered through Celite and washed with methanol-water $(40 \mathrm{~mL}: 10 \mathrm{~mL})$. The methanol was evaporated off, the aqueous layer saturated with solid $\mathrm{K}_{2} \mathrm{CO}_{3}$ and extracted with $\mathrm{CHCl}_{3}-\mathrm{MeOH}(9: 1)$ $(2 \times 20 \mathrm{~mL})$. The organic phase was dried $\left(\mathrm{MgSO}_{4}\right)$, and activated $\mathrm{MnO}_{2}(200 \mathrm{mg})$ was added and $\mathrm{O}_{2}$ was bubbled through the solution at $\mathrm{rt}$ for $30 \mathrm{~min}$. The mixture was then filtered, evaporated and the residue was purified by flash column chromatography $\left(\mathrm{CHCl}_{3}-\mathrm{MeOH}\right)$ to give the title compounds.

3-(3-Aminophenyl)-2,2,5,5-tetramethyl-2,5-dihydro-1 $\mathrm{H}$-pyrrol1-yloxyl radical (7). $404 \mathrm{mg}(35 \%), \mathrm{mp} 105-107{ }^{\circ} \mathrm{C} . R_{\mathrm{f}}: 0.30$ $\left(\mathrm{CHCl}_{3}-\mathrm{Et}_{2} \mathrm{O}, 2\right.$ : 1). Anal. calc. for $\mathrm{C}_{14} \mathrm{H}_{19} \mathrm{~N}_{2} \mathrm{O}$ : C 72.69, $\mathrm{H}$ 8.28, N 12.11; found: C 72.56, H 8.22, N 12.01\%. MS (EI): $\mathrm{m} / z$ (\%) $231\left(\mathrm{M}^{+}, 70\right), 216$ (18), 201 (67), 186 (100), 158 (77).

3-[4-(4-Aminobenzyl)piperazin-1-ylmethyl]-2,2,5,5-tetramethyl2,5-dihydro-1 $\boldsymbol{H}$-pyrrol-1-yloxyl radical (8). $737 \mathrm{mg} \mathrm{(43 \% ),} \mathrm{mp}$ $112-115{ }^{\circ} \mathrm{C} . R_{\mathrm{f}}: 0.10\left(\mathrm{CHCl}_{3}-\mathrm{MeOH}, 9\right.$ : 1). Anal. calc. for $\mathrm{C}_{20} \mathrm{H}_{31} \mathrm{~N}_{4} \mathrm{O}$ : C 69.92, $\mathrm{H}$ 9.10, N 16.32; found: C 69.90, H 9.05, $\mathrm{N} \mathrm{16.19 \%}$. MS (EI): $m / z(\%) 343\left(\mathrm{M}^{+}, 22\right), 313$ (17), 222 (26), 207 (84), 106 (100).

3-[4-(4-Nitrobenzyl)piperazin-1-ylmethyl]-2,2,5,5-tetramethyl2,5-dihydro-1 $\boldsymbol{H}$-pyrrol-1-yloxyl radical (22). A solution of compound 21 (2.38 g, $10.0 \mathrm{mmol})$, 4-nitrobenzyl bromide $(2.16 \mathrm{~g}, 10.0 \mathrm{mmol})$ and $\mathrm{K}_{2} \mathrm{CO}_{3}(1.38 \mathrm{~g}, 10.0 \mathrm{mmol})$ in $\mathrm{CHCl}_{3}$ $(30 \mathrm{~mL})$ was stirred and refluxed for $4 \mathrm{~h}$. After cooling the inorganic salt was filtered off, the organic phase was washed with water $(10 \mathrm{~mL})$, separated, dried $\left(\mathrm{MgSO}_{4}\right)$, filtered and evaporated. After flash column purification of the residue compound 22 was received as a yellow solid $2.53 \mathrm{~g}(68 \%), \mathrm{mp}$ 107-108 ${ }^{\circ} \mathrm{C}$. $R_{\mathrm{f}}: 0.48\left(\mathrm{CHCl}_{3}-\mathrm{MeOH}, 9\right.$ : 1). Anal. calc. for $\mathrm{C}_{20} \mathrm{H}_{29} \mathrm{~N}_{4} \mathrm{O}_{3}$ : C 64.30, H 7.87, N 15.01; found: C 64.25, H 7.71, N 14.91\%. MS (EI): $m / z$ (\%) $373\left(\mathrm{M}^{+}, 65\right), 343(14), 234$ (100), 221 (91), $136(33)$.

\section{General procedure for synthesis of compounds $12 \mathrm{C}-18 \mathrm{C}$}

To a stirred solution of amine 5-11 $(3.0 \mathrm{mmol})$ and $\mathrm{Et}_{3} \mathrm{~N}$ (666 mg, $6.6 \mathrm{mmol})$ in $\mathrm{CH}_{2} \mathrm{Cl}_{2}(20 \mathrm{~mL})$ was added dropwise a solution of freshly prepared 2-(chloroseleno)benzoyl chloride $4(762 \mathrm{mg}, 3.0 \mathrm{mmol})$ in $\mathrm{CH}_{2} \mathrm{Cl}_{2}(10 \mathrm{~mL})$ over $5 \mathrm{~min}$ at $\mathrm{rt}$ and the mixture was stirred for a further $1 \mathrm{~h}$. The organic phase was washed with brine $(10 \mathrm{~mL})$, dried $\left(\mathrm{MgSO}_{4}\right)$, filtered, evaporated and the residue was purified by flash column chromatography $\left(\mathrm{CHCl}_{3}-\mathrm{Et}_{2} \mathrm{O}\right.$ or $\left.\mathrm{CHCl}_{3}-\mathrm{MeOH}\right)$ to give compounds $\mathbf{1 2 C}-\mathbf{1 8 C}$ as orange-yellow solids in $35-73 \%$ yield.

2-[(1-Oxyl-2,2,5,5-tetramethyl-2,5-dihydro- $1 H$-pyrrol-3carboxamidoprop)-3yl]benzo $[d]$ isoselenazol-3-one radical (12C). Yellow solid $541 \mathrm{mg}(45 \%), \mathrm{mp} 126-128{ }^{\circ} \mathrm{C}$. $R_{\mathrm{f}}: 0.46\left(\mathrm{CHCl}_{3}-\right.$ $\mathrm{MeOH}, 9$ : 1). Anal. calc. for $\mathrm{C}_{19} \mathrm{H}_{24} \mathrm{~N}_{3} \mathrm{O}_{3}$ Se: C 54.16, H 5.74, N 9.97; found: C 54.02, H 5.68, N 9.82\%. MS (EI): $m / z(\%)$ $424 / 422 / 420 / 419 / 418 / 416 \quad\left(\mathrm{M}^{+}, \quad 0.2 / 1 / 0.5 / 0.2 / 0.2 / 0.02\right)$, 394/392/390/389/388/386 (1/7/3/1/1/0.1), 186/184/182/ 181/180/179 (8/43/21/7/8/1), 136 (100). ${ }^{77}$ Se NMR: 882 ppm.

2-[3-(1-Oxyl-2,2,5,5-tetramethyl-2,5-dihydro- $1 \mathrm{H}$-pyrrol-3yl)phenyl|benzo $[d]$ isoselenazol-3-one radical (14C). Yellow solid, $432 \mathrm{mg}(35 \%), \mathrm{mp} 148-150{ }^{\circ} \mathrm{C}$. $R_{\mathrm{f}}: 0.57\left(\mathrm{CHCl}_{3}-\right.$ $\mathrm{Et}_{2} \mathrm{O}, 2$ : 1). Anal. calc. for $\mathrm{C}_{21} \mathrm{H}_{21} \mathrm{~N}_{2} \mathrm{O}_{2}$ Se: C 61.17, H 5.13, $\mathrm{N}$ 6.79; found: C 61.10, H 5.12, N 6.67\%. MS (EI): $\mathrm{m} / \mathrm{z}$ (\%) 415/413/411/410/409/407 (M+ 2/9/4/1/2/0.3), 385/ 383/381/380/379/377 (4/20/9/3/4/0.4), 199 (100), 184 (47).

2- $\{$ 4-[1-(1-Oxyl-2,2,5,5-tetramethyl-2,5-dihydro-1 $H$-pyrrol3-ylmethyl)piperazin-4-yl]phenylmethyl $\}$ benzo $[d]$ isoselenazol-3one radical (15C). Yellow solid, $880 \mathrm{mg} 56 \%, \mathrm{mp} 115-117^{\circ} \mathrm{C}$. $R_{\mathrm{f}}: 0.34\left(\mathrm{CHCl}_{3}-\mathrm{MeOH}, 9: 1\right)$. Anal. calc. for $\mathrm{C}_{27} \mathrm{H}_{33} \mathrm{~N}_{4} \mathrm{O}_{2} \mathrm{Se}: \mathrm{C}$ 54.16, H 5.74, N 9.97; found: C 54.02, H 5.68, N 9.82\%. MS (EI): $m / z(\%) 527 / 525 / 523 / 522 / 521 / 519\left(\mathrm{M}^{+}, 0.2 / 1 / 0.5 / 0.2 / 0.2 /\right.$ 0.02), 497/495/493/492/491/489 (0.7/4/2/0.6/0.7/0.1), 290/ 288/286/285/284/282 (7/40/19/6/7/1), 136 (72), $122(100)$.

2-(1-Oxyl-2,2,5,5-tetramethyl-2,5-dihydro- $1 H$-pyrrol-3ylmethyl) benzo $[d]$ isoselenazol-3-one radical (16C). Pale yellow solid, $854 \mathrm{mg}(61 \%), \mathrm{mp} 165-166{ }^{\circ} \mathrm{C}$. $R_{\mathrm{f}}: 0.32\left(\mathrm{CHCl}_{3}-\right.$ $\mathrm{Et}_{2} \mathrm{O}, 2$ : 1). Anal. calc. for $\mathrm{C}_{16} \mathrm{H}_{19} \mathrm{~N}_{2} \mathrm{O}_{2}$ Se: C 54.86, H 5.47, $\mathrm{N}$ 8.00; found: C 54.71, H 5.44, N 7.93\%. MS (EI): $\mathrm{m} / z$ (\%) 353/351/349/348/347/345 ( $\left.\mathrm{M}^{+}, 0.7 / 4 / 2 / 0.6 / 0.7 / 0.1\right)$, 323/321/319/318/317/315 (1.5/8/4/1/1.5/0.2), 138 (41), 122 (62), 107 (100).

2-(1-Oxyl-3-carbomethoxy-2,2,5,5-tetramethylpyrrolidine-4ylmethyl) benzo $[d]$ isoselenazol-3-one radical (17C). Orange solid, $492 \mathrm{mg}(40 \%), \mathrm{mp} 207-209{ }^{\circ} \mathrm{C}$. $R_{\mathrm{f}}: 0.26\left(\mathrm{CHCl}_{3}-\right.$ $\mathrm{MeOH}, 9$ : 1). Anal. calc. for $\mathrm{C}_{18} \mathrm{H}_{23} \mathrm{~N}_{2} \mathrm{O}_{4} \mathrm{Se}: \mathrm{C} 52.42, \mathrm{H}$ 5.87, N 6.80; found: C 52.22, H 5.84, N 6.61\%. MS (EI): $m / z(\%)$ 413/411/409/408/407/405 ( $\left.\mathrm{M}^{+}, 1 / 5 / 3 / 0.8 / 1 / 0.1\right)$, $383 / 381 / 379 / 378 / 377 / 375(0.7 / 4 / 2 / 0.6 / 0.7 / 0.1), 269 / 267 /$ 265/264/263/261 (7/40/19/6/7/1), 201/199/197/196/195/ $193(18 / 100 / 47 / 15 / 18 / 2)$.

2-(1-Oxyl-2,2,6,6-tetramethylpiperidin-4yl) benzo $[d]$ isoselenazo3-one radical (18C). Orange-pink solid, $770 \mathrm{mg}(73 \%), \mathrm{mp}$ 230-231 ${ }^{\circ} \mathrm{C}$. $R_{\mathrm{f}}: 0.44\left(\mathrm{CHCl}_{3}-\mathrm{Et}_{2} \mathrm{O}, 2\right.$ : 1$)$. Anal. calc. for $\mathrm{C}_{16} \mathrm{H}_{21} \mathrm{~N}_{2} \mathrm{O}_{2}$ Se: C 54.38, H 5.99, N 7.93; found: C 54.30, H 5.88, $\mathrm{N} 7.85 \%$. MS (EI): $m / z(\%) 355 / 353 / 351 / 350 / 349 / 347\left(\mathrm{M}^{+}\right.$, $1 / 5 / 3 / 0.8 / 1 / 0.1), \quad 186 / 184 / 182 / 181 / 180 / 178 \quad(4 / 20 / 10 / 3 /$ 4/0.4), 140 (100).

\section{General procedure for synthesis of compounds 12B, 13B, 15B,} $18 B$

A solution of compound $12 \mathrm{C}, 13 \mathrm{C}, 15 \mathrm{C}$ or $18 \mathrm{C}(0.5 \mathrm{mmol})$ was refluxed with EtOH $(15 \mathrm{~mL}$ ) (saturated with $\mathrm{HCl}$ ) for $20 \mathrm{~min}$. After cooling, the solvent was evaporated off and the residue was crystallized with acetone or $\mathrm{Et}_{2} \mathrm{O}$ to give title compounds as off-white or yellow solids in $68-82 \%$ yield.

2-[(1-Hydroxy-2,2,5,5-tetramethyl-2,5-dihydro-1 $H$-pyrrol-3carboxamidoprop)-3yl]benzo $[d]$ isoselenazol-3-one hydrochloride (12B). Off-white solid $160 \mathrm{mg}(70 \%)$, mp 115-117 ${ }^{\circ} \mathrm{C}$. Anal. calc. for $\mathrm{C}_{19} \mathrm{H}_{26} \mathrm{ClN}_{3} \mathrm{O}_{3} \mathrm{Se}$ : C 49.74, $\mathrm{H}$ 5.71, N 9.16; found: $\mathrm{C}$ 49.72, H 5.70, N 8.99\%. ${ }^{1} \mathrm{H}$ NMR (400 MHz, $\left.\mathrm{D}_{2} \mathrm{O}\right): \delta 7.76$ $(\mathrm{m}, 2 \mathrm{H}$, ar $\mathrm{CH}), 7.56(\mathrm{t}, J=6 \mathrm{~Hz}, 1 \mathrm{H}, \operatorname{ar} \mathrm{CH}), 7.39(\mathrm{t}, J=$ $6 \mathrm{~Hz}, 1 \mathrm{H}$, ar $\mathrm{CH}), 6.05(\mathrm{~s}, 1 \mathrm{H}$, olephinic $\mathrm{CH}), 3.81(\mathrm{t}, J=$ $\left.6.4 \mathrm{~Hz}, 2 \mathrm{H}, \mathrm{NCH}_{2}\right), 3.26\left(\mathrm{t}, J=6 \mathrm{~Hz}, 2 \mathrm{H}, \mathrm{NCH}_{2}\right), 1.92(\mathrm{~m}, 2 \mathrm{H}$, $\left.\mathrm{CH}_{2}-\mathrm{CH}_{2}-\mathrm{CH}_{2}\right), 1.45\left(\mathrm{~s}, 6 \mathrm{H}, \mathrm{CCH}_{3}\right), 1.32\left(\mathrm{~s}, 6 \mathrm{H}, \mathrm{CCH}_{3}\right) \cdot{ }^{13} \mathrm{C}$ NMR (100.5 MHz, $\left.\mathrm{D}_{2} \mathrm{O}\right): \delta 168.7,164.3,139.5,137.1,135.1$, $132.8,127.8,127.1,126.8,125.0,78.6,76.0,43.41,37.65,28.6$, 23.3 br, 22.07 br. ${ }^{77} \mathrm{Se}$ NMR $\left(\mathrm{CDCl}_{3}-\mathrm{MeOH}\right): \delta 1043$; ( $\left.\mathrm{D}_{2} \mathrm{O}\right): \delta$ $925 \mathrm{ppm}$.

2-[3-(1-Hydroxy-2,5,5-trimethylpirrolidin-2yl)phenyl|benzo[d]isoselenazol-3-one hydrochloride (13B). White solid $148 \mathrm{mg}$ $(68 \%), \mathrm{mp} 141-142^{\circ} \mathrm{C}$. Anal. calc. for $\mathrm{C}_{20} \mathrm{H}_{23} \mathrm{ClN}_{2} \mathrm{O}_{2} \mathrm{Se}$ : $\mathrm{C}$ 54.87, H 5.29, N 6.40; found: C 54.73, H 5.20, N 6.22\%. ${ }^{1} \mathrm{H}$ NMR (400 MHz, DMSO-d $\left.{ }_{6}\right): \delta 8.14(\mathrm{~d}, J=7.7 \mathrm{~Hz}, 1 \mathrm{H}$, ar $\mathrm{CH}), 7.88(\mathrm{~d}, J=8.0 \mathrm{~Hz}, 1 \mathrm{H}$, ar CH), $7.87(\mathrm{~s}, 1 \mathrm{H}, \operatorname{ar} \mathrm{CH}), 7.66$ $\left(\mathrm{dd}, J_{1}=7.7 \mathrm{~Hz}, J_{2}=7.4 \mathrm{~Hz}, 2 \mathrm{H}, \operatorname{ar} \mathrm{CH}\right), 7.56(\mathrm{~d}, J=7.7 \mathrm{~Hz}$, $1 \mathrm{H}$, ar $\mathrm{CH}), 7.51(\mathrm{~d}, J=7.5 \mathrm{~Hz}, 1 \mathrm{H}$, ar $\mathrm{CH}), 7.47\left(\mathrm{dd}, J_{1}=\right.$ $7.5 \mathrm{~Hz}, J_{2}=7.4 \mathrm{~Hz}, 2 \mathrm{H}$, ar $\mathrm{CH}, 2.58\left(\mathrm{~m}, 1 \mathrm{H}, \mathrm{CH}_{2}\right), 2.37(\mathrm{~m}$, $\left.1 \mathrm{H}, \mathrm{CH}_{2}\right), 2.21\left(\mathrm{~m}, 1 \mathrm{H}, \mathrm{CH}_{2}\right), 2.04\left(\mathrm{~m}, 1 \mathrm{H}, \mathrm{CH}_{2}\right), 1.63(\mathrm{~s}, 3 \mathrm{H}$, $\left.\mathrm{CCH}_{3}\right), 1.41\left(\mathrm{~s}, 3 \mathrm{H}, \mathrm{CCH}_{3}\right), 1.28$ (br s, $\left.3 \mathrm{H}, \mathrm{CCH}_{3}\right) .{ }^{13} \mathrm{C} \mathrm{NMR}$ $\left(100.5 \mathrm{MHz}, \mathrm{D}_{2} \mathrm{O}\right): \delta 168.1,140.0,138.3,133.3,130.4,128.3$, $127.4,127.0,126.6,125.0,76.6,75.1,35.1,31.4 \mathrm{ppm} .{ }^{77} \mathrm{Se} \mathrm{NMR}$ (76.3 MHz, DMSO-d ${ }_{6}$ ): $\delta 891 \mathrm{ppm}$.

2-\{4-[1-(1-Hydroxy-2,2,5,5-tetramethyl-2,5-dihydro- $1 \mathrm{H}$-pyrrol3-ylmethyl)piperazin-4-yl]phenylmethyl $\}$ benzo[ $d]$ isoselenazol-3one trihydrochloride (15B). White solid, $286 \mathrm{mg}(82 \%), \mathrm{mp}$ 195-197 ${ }^{\circ} \mathrm{C}$. Anal. calc. for $\mathrm{C}_{27} \mathrm{H}_{37} \mathrm{Cl}_{3} \mathrm{~N}_{4} \mathrm{O}_{2}$ Se: C 51.08, H 5.87, 
N 8.82; found: C 51.03, H 5.79, N 8.70\%. ${ }^{1} \mathrm{H}$ NMR (400 MHz, $\left.\mathrm{D}_{2} \mathrm{O}\right): \delta 7.96(\mathrm{~d}, J=7.6 \mathrm{~Hz}, 1 \mathrm{H}, \operatorname{ar} \mathrm{CH}), 7.89(\mathrm{~d}, J=7.2 \mathrm{~Hz}$, $1 \mathrm{H}$, ar $\mathrm{CH}), 7.72\left(\mathrm{dd}, J_{1}=7.6 \mathrm{~Hz}, J_{2}=7.2 \mathrm{~Hz}, 1 \mathrm{H}\right.$, ar $\left.\mathrm{CH}\right)$, 7.59 (br s, $4 \mathrm{H}$, ar CH), $7.53\left(\mathrm{dd}, J_{1}=7.6 \mathrm{~Hz}, J_{2}=7.2 \mathrm{~Hz}, 1 \mathrm{H}\right.$, ar $\mathrm{CH}), 5.84(\mathrm{~s}, 1 \mathrm{H}$, olephinic $\mathrm{CH}), 4.41\left(\mathrm{~s}, 2 \mathrm{H}, \mathrm{NCH}_{2}\right), 4.35$ (s, $2 \mathrm{H}, \mathrm{NCH}_{2}$ ), 3.51 (s, $2 \mathrm{H}, \mathrm{NCH}_{2}$ ), 3.34 (brs, $\left.2 \mathrm{H}, \mathrm{NCH}_{2}\right), 1.47$ (s, $\left.6 \mathrm{H}, \mathrm{CCH}_{3}\right), 1.45\left(\mathrm{~s}, 6 \mathrm{H}, \mathrm{CCH}_{3}\right) .{ }^{13} \mathrm{C}$ NMR $\left(100.5 \mathrm{MHz}, \mathrm{D}_{2} \mathrm{O}\right)$ : $\delta 167.9,140.1,133.3,132.7,127.0,126.8,125.0,78.5,76.8,57.7$, 49.6, 49.0, 24.2 br, 22.9 ppm. ${ }^{77} \mathrm{Se} \mathrm{NMR}\left(76.3 \mathrm{MHz}, \mathrm{D}_{2} \mathrm{O}\right): \delta$ 997 ppm.

2-(1-Hydroxy-2,2,6,6-tetramethylpiperidin-4-yl) benzo $[d]-$ isoselenazol-3-one radical hydrochloride (18B). Yellow solid, $144 \mathrm{mg}(74 \%)$, mp: $205-206^{\circ} \mathrm{C}$. Anal. calc. for $\mathrm{C}_{16} \mathrm{H}_{23} \mathrm{ClN}_{2} \mathrm{O}_{2} \mathrm{Se}$ : C 49.30, H 5.95, N 7.19; found: C 49.22, H 5.92, N 7.03\%. ${ }^{1} \mathrm{H}$ NMR (400 MHz, $\left.\mathrm{D}_{2} \mathrm{O}\right): \delta 7.87(\mathrm{~d}, J=7.6 \mathrm{~Hz}, 1 \mathrm{H}$, ar $\mathrm{CH})$, $7.82\left(\mathrm{~d}, J=8.0 \mathrm{~Hz}, 1 \mathrm{H}\right.$, ar CH), $7.62\left(\mathrm{dd}, J_{1}=8.0 \mathrm{~Hz}, J_{2}=\right.$ $7.2 \mathrm{~Hz}, 1 \mathrm{H}$, ar CH), $7.46\left(\mathrm{dd}, J_{1}=7.6 \mathrm{~Hz}, J_{2}=7.2 \mathrm{~Hz}, 1 \mathrm{H}\right.$, ar $\mathrm{CH}), 4.93(\mathrm{~m}, 1 \mathrm{H}, \mathrm{NCH}), 2.30(\mathrm{~m}, 2 \mathrm{H}, \mathrm{CHH}), 2.10(\mathrm{n}, 2 \mathrm{H}$, $\mathrm{CH} H), 1.52\left(\mathrm{~s}, 6 \mathrm{H}, \mathrm{CCH}_{3}\right), 1.43\left(\mathrm{~s}, 6 \mathrm{H}, \mathrm{CCH}_{3}\right) .{ }^{13} \mathrm{C} \mathrm{NMR}$ $\left(100.5 \mathrm{MHz}, \mathrm{D}_{2} \mathrm{O}\right): \delta 168.7,139.5,132.9,127.8,127.4,126.9$, 125.0, 68.8, 45.0, 42.4, 27.3, 19.6 ppm. ${ }^{77} \mathrm{Se} \mathrm{NMR}(76.3 \mathrm{MHz}$, $\left.\mathrm{D}_{2} \mathrm{O}\right): \delta 890 \mathrm{ppm}$.

\section{General procedure for synthesis of compounds 12A-18A}

To a solution of nitroxide $\mathbf{1 2 C}-\mathbf{1 8 C}(1.0 \mathrm{mmol})$ in $\mathrm{AcOH}(8 \mathrm{~mL})$ Fe powder $(560 \mathrm{mg}, 10 \mathrm{mmol})$ was added and the mixture was warmed up to $70{ }^{\circ} \mathrm{C}$ until the reaction started. The mixture was stirred at room temperature for $1 \mathrm{~h}$, diluted with water $(15 \mathrm{~mL})$, decanted and the decanted aqueous solution made alkaline with solid $\mathrm{K}_{2} \mathrm{CO}_{3}$. The mixture was extracted with $\mathrm{CHCl}_{3}(3 \times 15 \mathrm{~mL})$, dried $\left(\mathrm{MgSO}_{4}\right)$, filtered, evaporated and after chromatographic purification $\left(\mathrm{CHCl}_{3}-\mathrm{MeOH}\right)$ we got the title amines 12A-18A in $47-59 \%$ yield.

2,2'-Diselenobis $[N$-(2,2,5,5-tetramethyl-2,5-dihydro- $1 H$-pyrrol3-carboxamido-prop-3-yl)|benzamide (12A). Beige solid, $199 \mathrm{mg}(49 \%)$, mp $182-183{ }^{\circ} \mathrm{C}\left(2 \mathrm{HCl}\right.$ salt). $R_{\mathrm{f}}$ : $0.16(\mathrm{MeOH})$. Anal. calc. for $\mathrm{C}_{38} \mathrm{H}_{54} \mathrm{Cl}_{2} \mathrm{~N}_{6} \mathrm{O}_{4} \mathrm{Se}_{2}$ : C 51.42, H 6.13, N 9.47; found: C 51.30, H 6.00, N 9.31\%. ${ }^{1} \mathrm{H}$ NMR (400 MHz, $\left.\mathrm{D}_{2} \mathrm{O}\right): \delta$ $7.47(\mathrm{~d}, J=7.6 \mathrm{~Hz}, 2 \mathrm{H}), 7.48(\mathrm{~d}, J=7.6 \mathrm{~Hz}, 2 \mathrm{H}), 7.03\left(\mathrm{dd}, J_{1}=\right.$ $\left.7.6 \mathrm{~Hz}, J_{2}=7.2 \mathrm{~Hz}, 2 \mathrm{H}\right), 6.97\left(\mathrm{dd}, J_{1}=7.6 \mathrm{~Hz}, J_{2}=7.2 \mathrm{~Hz}\right.$, $2 \mathrm{H}), 6.15$ (s, 2H), 3.31 (br dd, 4H), 3.22 (br dd, 4H), 1.75 (br $\mathrm{dd}, 4 \mathrm{H}), 1.53(\mathrm{~s}, 6 \mathrm{H}), 1.41(\mathrm{~s}, 6 \mathrm{H}) \cdot{ }^{13} \mathrm{C}$ NMR $(100.5 \mathrm{MHz}$, $\left.\mathrm{D}_{2} \mathrm{O}\right): \delta 170.0,164.6,138.1,136.6,133.6,132.2,131.3,127.9$, 127.2, 71.8, 68.8, 37.8, 37.3, 28.3, 26.5, 26.2 ppm. ${ }^{77} \mathrm{Se}$ NMR $\left(\mathrm{CDCl}_{3}-\mathrm{MeOH}\right): \delta 442 \mathrm{ppm}$; $\left(\mathrm{D}_{2} \mathrm{O}\right): \delta 449 \mathrm{ppm}$.

2,2' -Diselenobis[ $N$-3-(2,5,5-trimethylpyrrolidin-2-yl)phenyl]benzamide oxalate (13A). White solid, $474 \mathrm{mg}(55 \%), \mathrm{mp}$ 161-164 ${ }^{\circ} \mathrm{C}$. $R_{\mathrm{f}}: 0.11\left(\mathrm{CHCl}_{3}-\mathrm{MeOH}, 2\right.$ : 1). Anal. calc. for $\mathrm{C}_{42} \mathrm{H}_{48} \mathrm{~N}_{4} \mathrm{O}_{6} \mathrm{Se}_{2}$ : C 58.47, H 5.61, N 6.49; found: C 58.33, $\mathrm{H}$ 5.87, N 6.40\%. ${ }^{1} \mathrm{H}$ NMR (for base) (400 MHz, $\left.\mathrm{CDCl}_{3}\right): \delta 9.9$ (br s, 2H, NH), 7.92 (s, 2H, ar CH), $7.80(\mathrm{~d}, J=6.4 \mathrm{~Hz}, 2 \mathrm{H}$, ar $\mathrm{CH}), 7.76(\mathrm{~d}, J=7.6 \mathrm{~Hz}, 2 \mathrm{H}$, ar $\mathrm{CH}), 7.22-7.28(\mathrm{~m}, 4 \mathrm{H}$, ar $\mathrm{CH}), 7.11-7.18(\mathrm{~m}, 6 \mathrm{H}$, ar $\mathrm{CH}), 2.44-2.52\left(\mathrm{~m}, 2 \mathrm{H}, \mathrm{CH}_{2}\right)$, 2.08-2.18 (m, 2H, $\left.\mathrm{CH}_{2}\right), 1.95-2.02\left(\mathrm{~m}, 2 \mathrm{H}, \mathrm{CH}_{2}\right), 1.82-1.90$ (m, $\left.2 \mathrm{H}, \mathrm{CH}_{2}\right), 1.69\left(\mathrm{~s}, 6 \mathrm{H}, \mathrm{CCH}_{3}\right), 1.21\left(\mathrm{~s}, 6 \mathrm{H}, \mathrm{CCH}_{3}\right), 1.12(\mathrm{~s}, 6 \mathrm{H}$, $\left.\mathrm{CCH}_{3}\right) .{ }^{13} \mathrm{C}$ NMR $\left(100.5 \mathrm{MHz}, \mathrm{CDCl}_{3}\right): \delta 167.2,143.5,139.2$, $133.9,133.3,132.0,131.3,129.4,128.6,126.3,121.7,120.8$, $118.7,70.05,65.0,38.3,36.4,29.20,29.15,28.6 .{ }^{77} \mathrm{Se}$ NMR $\left(\mathrm{D}_{2} \mathrm{O}\right): \delta 455 \mathrm{ppm}$.

2,2' -Diselenobis $\mathrm{N}$-3-(2,2,5,5-tetramethyl-2,5-dihydro- $1 \mathrm{H}$ pyrrol-3-yl)phenyl)|benzamide (14A). Yellow solid, $398 \mathrm{mg}$ (50\%), mp $217-220{ }^{\circ} \mathrm{C} . R_{\mathrm{f}}: 0.50\left(\mathrm{CHCl}_{3}-\mathrm{MeOH}, 2\right.$ : 1). Anal. calc. for $\mathrm{C}_{42} \mathrm{H}_{46} \mathrm{~N}_{4} \mathrm{O}_{2} \mathrm{Se}_{2}$ : C 63.31, H 5.85, N 7.03; found: C 63.27, H 5.76, N 7.00\%. ${ }^{1} \mathrm{H}$ NMR (400 MHz, DMSO-d ${ }_{6}$ ): $\delta 8.51$ $(\mathrm{d}, J=8.0 \mathrm{~Hz}, 1 \mathrm{H}), 7.82(\mathrm{~d}, J=6.8 \mathrm{~Hz}, 1 \mathrm{H}), 7.78(\mathrm{~s}, 1 \mathrm{H}), 7.57$ $(\mathrm{m}, 1 \mathrm{H}), 7.50(\mathrm{~m}, 1 \mathrm{H}), 7.40(\mathrm{~m}, 2 \mathrm{H}), 7.22(\mathrm{~d}, J=7.2 \mathrm{~Hz}, 1 \mathrm{H})$, 6.07 (s, 1H), $2.90(\mathrm{~s}, 1 \mathrm{H}), 1.65$ (s, 6H), $1.54(\mathrm{~s}, 6 \mathrm{H}) .{ }^{13} \mathrm{C} \mathrm{NMR}$
(100.5 MHz, DMSO-d $\left.{ }_{6}\right): \delta 165.1,142.9,140.9,139.9,132.9$, $131.5,131.3,129.9,129.3,127.3,127.1,125.7,124.0,123.8$, 122.8, 70.5, 66.7, $27.1 \mathrm{ppm} .{ }^{77} \mathrm{Se}$ NMR (DMSO-d 6 ): $\delta 448 \mathrm{ppm}$.

2,2' -Diselenobis $[N-\{4-[1-(2,2,5,5-$ tetramethyl-2,5-dihydro$1 \mathrm{H}$-pyrrol-3-ylmethyl)piperazin-4-yl|phenylmethyl $\}$ |benzamide (15A). Yellow solid, $479 \mathrm{mg}(47 \%), \mathrm{mp} 112-115{ }^{\circ} \mathrm{C} . R_{\mathrm{f}}$ : $0.20\left[\mathrm{MeOH}-\mathrm{NH}_{4} \mathrm{OH}\right.$ (aq. $25 \%$ ), 40 : 1]. Anal. calc. for $\mathrm{C}_{54} \mathrm{H}_{70} \mathrm{~N}_{8} \mathrm{O}_{2} \mathrm{Se}_{2}$ : C 63.52, H 6.91, N 10.97; found: $\mathrm{C} 63.35, \mathrm{H}$ 7.02, N 10.88\%. ${ }^{1} \mathrm{H}$ NMR (400 MHz, DMSO-d 6 ): $\delta 10.5$ (br, $2 \mathrm{H}), 7.92(\mathrm{~d}, J=6.0 \mathrm{~Hz}, 2 \mathrm{H}), 7.78(\mathrm{br}, 2 \mathrm{H}), 7.67(\mathrm{~d}, J=6.8 \mathrm{~Hz}$, $2 \mathrm{H}), 7.39(\mathrm{~m}, 4 \mathrm{H}), 7.26(\mathrm{~m}, 4 \mathrm{H}), 5.36(\mathrm{~s}, 2 \mathrm{H}), 3.49(\mathrm{br}, 8 \mathrm{H}), 3.41$ (s, 4H), $2.83(\mathrm{~s}, 4 \mathrm{H}), 2.35(\mathrm{br}, 8 \mathrm{H}) 1.13(\mathrm{~s}, 6 \mathrm{H}), 1.10(\mathrm{~s}, 6 \mathrm{H}) \cdot{ }^{13} \mathrm{C}$ NMR (100.5 MHz, DMSO-d ${ }_{6}$ ): $\delta 166.1,142.9,137.5$ br, 133.8 br, $133.2,131.9,129.1,128.5,126.3,120.3,65.9,62.6,61.6$, 54.9, 53.0, 52.6, 45.6, 30.9, 29.7, 21.7, 11.6 ppm. ${ }^{77} \mathrm{Se} \mathrm{NMR}$ $\left(\right.$ DMSO-d $\left.{ }_{6}\right): \delta 448$ ppm.

2,2'-Diselenobis $[N$-(2,2,5,5-tetramethyl-2,5-dihydro- $1 H$-pyrrol3-ylmethyl)]benzamide (16A). White solid, $349 \mathrm{mg}$ (52\%), $\mathrm{mp}$ 196-197 ${ }^{\circ} \mathrm{C}$. $R_{\mathrm{f}}: 0.87\left(\mathrm{MeOH}-\mathrm{NH}_{4} \mathrm{OH}, 40: 1\right)$. Anal. calc. for $\mathrm{C}_{32} \mathrm{H}_{42} \mathrm{~N}_{4} \mathrm{O}_{2} \mathrm{Se}_{2}$ : C 57.14, H 6.29, N 8.33; found: C 57.11, H $6.15, \mathrm{~N} 8.20 \%$. ${ }^{1} \mathrm{H}$ NMR (400 MHz, DMSO-d $\left.{ }_{6}\right): \delta 8.90(\mathrm{br}$, $2 \mathrm{H}), 7.80(\mathrm{~d}, J=6.4 \mathrm{~Hz}, 2 \mathrm{H}), 7.68(\mathrm{~d}, J=6.8 \mathrm{~Hz}, 2 \mathrm{H}), 7.31$ (m, 4H), 5.40 (s, 2H), 3.93 (br s, 4H), 1.19 (s, 12H), 1.11 (s, $12 \mathrm{H}) .{ }^{13} \mathrm{C}$ NMR $\left(100.5 \mathrm{MHz}, \mathrm{DMSO}-\mathrm{d}_{6}\right): \delta 167.0,143.9,133.1$, 132.0, 131.4, 131.1, 129.8, 127.9, 126.1, 65.4, 62.6, 36.1, 31.1, $29.8 \mathrm{ppm} .{ }^{77} \mathrm{Se}$ NMR (DMSO-d $\left.{ }_{6}\right): \delta 447 \mathrm{ppm}$.

2,2'-Diselenobis[ $N$-(3-carbomethoxy-2,2,5,5-tetramethylpyrroli-

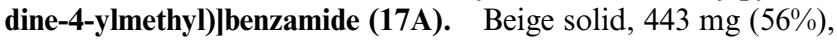
mp 207-209 ${ }^{\circ} \mathrm{C} . R_{\mathrm{f}}: 0.67\left(\mathrm{MeOH}-\mathrm{NH}_{4} \mathrm{OH}, 40: 1\right)$. Anal. calc. for $\mathrm{C}_{36} \mathrm{H}_{50} \mathrm{~N}_{4} \mathrm{O}_{6} \mathrm{Se}_{2}$ : C 54.54, H 6.36, N 7.07; found: C 54.50, H 6.20, N 6.95\%. ${ }^{1} \mathrm{H}$ NMR (400 MHz, $\left.\mathrm{CDCl}_{3}\right): \delta 7.85$ (d, $J=$ $8.0 \mathrm{~Hz}, 2 \mathrm{H}), 7.49(\mathrm{~d}, J=7.2 \mathrm{~Hz}, 2 \mathrm{H}), 7.20(\mathrm{~m}, 4 \mathrm{H}), 7.04$ (br s, $2 \mathrm{H}), 3.64\left(\mathrm{~s}, 6 \mathrm{H}, \mathrm{OCH}_{3}\right), 3.56-3.59(\mathrm{~m}, 2 \mathrm{H}), 3.36(\mathrm{~m}, 2 \mathrm{H}), 2.80$ $(\mathrm{d}, 2 \mathrm{H}), 2.51(\mathrm{~m}, 6 \mathrm{H}) 1.38(\mathrm{~s}, 6 \mathrm{H}), 1.28(\mathrm{~s}, 6 \mathrm{H}), 1.09(\mathrm{~s}, 6 \mathrm{H}), 1.04$ (s, 6H). ${ }^{13} \mathrm{C}$ NMR (100.5 MHz, $\mathrm{CDCl}_{3}$ ): $\delta 175.0,167.8,133.5$, $132.2,131.6,131.2,126.5,125.9,60.1,59.93,59.86,52.1,50.5$, 40.8, 31.7, 29.7, 27.4, $25.1 \mathrm{ppm} .{ }^{77} \mathrm{Se} \mathrm{NMR}\left(\mathrm{CDCl}_{3}\right): \delta 459.6$, $459.8 \mathrm{ppm}$ (because of diastereomers).

2,2' -Diselenobis $[N$-(2,2,6,6-tetramethylpiperidin-4-yl)]benzamide (18A). White solid, $354 \mathrm{mg}(49 \%), \mathrm{mp} 224-226{ }^{\circ} \mathrm{C} . R_{\mathrm{f}}$ : $0.35\left(\mathrm{MeOH}-\mathrm{NH}_{4} \mathrm{OH}, 40\right.$ : 1). Anal. calc. for $\mathrm{C}_{36} \mathrm{H}_{46} \mathrm{~N}_{4} \mathrm{O}_{2} \mathrm{Se}_{2}$ : C 59.66, H 6.40, N 7.73; found: C 54.60, H 6.38, N 7.67\%. ${ }^{1} \mathrm{H}$ NMR (400 MHz, DMSO-d $\left.{ }_{6}\right): \delta 8.42(\mathrm{br}, 2 \mathrm{H}), 8.0(\mathrm{~d}, J=$ $6.4 \mathrm{~Hz}, 2 \mathrm{H}), 7.68(\mathrm{~d}, J=6.8 \mathrm{~Hz}, 2 \mathrm{H}), 7.31(\mathrm{~m}, 4 \mathrm{H}), 5.40(\mathrm{~s}$, 2H), 3.93 (br s, 4H), 1.19 (s, 12H), 1.11 (s, 12H). ${ }^{13} \mathrm{C}$ NMR $\left(100.5 \mathrm{MHz}, \mathrm{DMSO}-\mathrm{d}_{6}\right): \delta 167.0,143.9,133.1,132.0,131.4$, $131.1,129.8,127.9,126.1,65.4,62.6,36.1,31.1,29.8 \mathrm{ppm} .{ }^{77} \mathrm{Se}$ NMR (DMSO-d d $_{6}: \delta 447 \mathrm{ppm}$.

\section{Acknowledgements}

This study was supported by the Hungarian Research Foundation (OTKA grant T048334 and M045190), the Deutsche Forschungsgemeinschaft (Si255/11-1) and partly by the Alexander von Humboldt-Stiftung in the form of a research fellowship to G. M. The authors thank to Dr J. Jek (ICN Hungary) for helpful discussion. H. S. is a Fellow of the National Foundation for Cancer Research (NFCR), Bethesda, MD, USA.

\section{References}

1 T. C. Stadtman, J. Biol. Chem., 1991, 266, 16257.

2 F. Ursini, in Oxidative Processes and Antioxidants, ed. R. Paoletti, New York, Raven Press, 1994, p. 25.

3 L. Flohé, E. A. Günzler and H. H. Schock, FEBS Lett., 1973, 32, 132.

4 J. T. Rotruck, A. L. Pope, H. E. Ganther, A. B. Swanson, D. G. Hafeman and W. G. Hoekstra, Science, 1973, 179, 588. 
5 M. Maiorino, K. D. Aumann, R. Brigelius-Flohé, D. Doria, J. van den Heuvel, J. McCarthy, A. Roveri, F. Ursini and L. Flohé, Biol. Chem. Hoppe-Seyler, 1995, 376, 651.

6 C. Rocher, J. L. Lalanne and J. Chaudière, Eur. J. Biochem., 1992, 205, 955 .

7 K. R. Maddipati and L. J. Marnett, J. Biol. Chem., 1987, 262, 17398.

8 F. F. Chu, J. H. Doroshow and R. S. Esworthy, J. Biol. Chem., 1993, 268, 2571.

9 R. Brigelius-Flohé, Free Radical Biol. Med., 1999, 27, 951.

10 C. Krebz-Remy, P. Mehlen, M. E. Mirault and A. P. Arrigo, J. Cell Biol., 1996, 133, 1083.

11 F. Weitzel and A. Wendel, J. Biol. Chem., 1993, 268, 6288.

12 G. Mugesh and H. B. Singh, Chem. Soc. Rev., 2000, 29, 347.

13 C. A. Collins, F. H. Fry, A. L. Holme, A. Yiakouvaki, A. Qenaei, C. Pourzand and C. Jacob, Org. Biomol. Chem., 2005, 3, 1541.

14 G. Mugesh, W. W. du Mont and H. Sies, Chem. Rev., 2001, 101, 2125.

15 A. Müller, E. Cadenas, P. Graf and H. Sies, Biochem. Pharmacol., 1984, 33, 3235 .

16 A. Wendel, M. Fausel, H. Safayhi, G. Tiegs and R. Otter, Biochem. Pharmacol., 1984, 33, 3241.

17 H. J. Reich and C. P. Japserse, J. Am. Chem. Soc., 1987, 109, 5549.

18 P. Chan, P. Cotelle, L. J. Bernier and J. P. Henichart, Bioorg. Med. Chem. Lett., 1991, 1, 277.

19 P. V. Jacquemin, L. E. Christiaens, M. J. Renson, M. J. Evers and N. Dereu, Tetrahedron Lett., 1992, 33, 3863.

20 J. Chaudière, J. C. Yadan, I. Erdelmeier, C. Tailhan-Lomont, and M. Moutet, in Oxidative Processes and Antioxidants, ed. R. Paoletti, New York, Raven Press, 1994, p. 165.

21 A. Mohsine and L. Christiaens, L., Heterocycles, 1996, 43, 2567.

22 T. G. Back and B. P. Dyck, J. Am. Chem. Soc., 1997, 119, 2079.

23 V. Galet, J. Bernier, J. Henichart, D. Lesieur, C. Abadie, L. Rochette, A. Lindenbaum, J. Chalas, J. Renaud, de la Faverie, B. Pfeiffer and P. Renard, J. Med. Chem., 1994, 37, 2903.

24 S. Ostrovidov, P. Franck, D. Joseph, L. Martarello, G. Kirsch, F. Belleville, P. Nabet and B. Dousset, J. Med. Chem., 200, 43, 1762.

25 I. A. Cotgreave, P. Moldeus, R. Brattsand, A. Hallberg, C. M. Andersson and L. Engman, Biochem. Pharmacol., 1992, 43, 793.

26 S. R. Wilson, P. A. Zucker, R. R. C. Huang and A. Spector, J. Am. Chem. Soc., 1989, 111, 5936.

27 M. Iwaoka and S. Tomoda, J. Am. Chem. Soc., 1994, 116, 2557.
28 T. Wirth, Molecules, 1998, 3, 164.

29 G. Mugesh, A. Panda, H. B. Singh, N. S. Punekar and R. J. Butcher, Chem. Commun., 1998, 2227.

30 G. Mugesh, A. Panda, H. B. Singh, N. S. Punekar and R. J. Butcher, J. Am. Chem. Soc., 2001, 123, 839.

31 H. Sies, Free Radical Biol. Med., 1993, 14, 313.

32 T. P. M. Akerboom, H. Sies and D. M. Ziegler, Arch. Biochem. Biophys., 1995, 316, 220.

33 J. Chaudière, O. Courtin and J. LeClaire, Arch. Biochem. Biophys. 1992, 296, 328.

34 H. O. Hankovszky, K. Hideg, I. Bódi and L. Frank, L., J. Med. Chem., 1986, 29, 1138.

35 M. C. Krishna, W. Degraff, H. O. Hankovszky, P. C. Sár, T. Kálai, J. Jek, A. Russo, J. B. Mitchell and K. Hideg, J. Med. Chem., 1998, 41, 3477.

36 R. A. Shankar, K. Hideg, J. L. Zweier and P. Kuppusamy, P., J. Pharmacol. Exp. Ther., 2000, 292, 838-845.

37 P. Twomey, J. Taira, W. DeGraff, J. B. Mitchell, A. Russo, M. C. Krishna, H. O. Hankovszky, L. Frank and K. Hideg, Free Radical Biol. Med., 1997, 22, 909.

38 S. Gadányi, T. Kálai, J. Jek, Z. Berente and K. Hideg, Synthesis, 2000, 2039.

39 R. Cantineau, G. Thiange, A. Pelenevaux, L. Christiaens and M. Guillaume, J. Labelled Compd. Radiopharm., 1986, 23, 59.

40 H. O. Hankovszky, K. Hideg, M. J. Lovas, G. Jerkovich, A. Rockenbauer, M. Gyr and P. Sohár, Can. J. Chem., 1989, 67, 1392.

41 H. O. Hankovszky, K. Hideg, K. and L. Lex, Synthesis, 1981, 147.

42 K. Hideg, H. O. Hankovszky, H. A. Halász and P. Sohár, J. Chem. Soc., Perkin Trans. 1., 1988, 2905.

43 E. G. Rozantsev, Free Nitroxyl Radicals, NewYork, Plenum Press, 1970.

44 T. Kálai, M. Balog, J. Jek, W. L. Hubbell and K. Hideg, Synthesis, 2002,2365

45 S. Ram and R. E. Ehrenkaufer, Synthesis, 1988, 91.

46 T. Kálai, H. O. Hankovszky, É. Hideg, J. Jek and K. Hideg, ARKIVOC, 2002, 3(iii), 112.

47 P. C. Sár, T. Kálai, M. N. Bárácz, G. Jerkovich and K. Hideg, Synth. Commun., 1995, 25, 2929.

48 H. Fischer and N. Dereu, Bull. Soc. Chim. Belg., 1987, 96, 757.

49 A. Roveri, M. Maiorino and F. Ursini, Methods Enzymol., 1994, 233, 202. 